\title{
A LOWER BOUND OF THE HYPERBOLIC DIMENSION FOR MEROMORPHIC FUNCTIONS HAVING A LOGARITHMIC HÖLDER TRACT
}

\author{
VOLKER MAYER
}

\begin{abstract}
We improve existing lower bounds of the hyperbolic dimension for meromorphic functions that have a logarithmic tract $\Omega$ which is a Hölder domain. These bounds are given in terms of the fractal behavior, measured with integral means, of the boundary of $\Omega$ at infinity.
\end{abstract}

\section{INTRODUCTION}

Let $f: \mathbb{C} \rightarrow \hat{\mathbb{C}}$ be a transcendental meromorphic function with Julia set $\mathcal{J}_{f}$. The different fractal dimensions of dynamically significant sets such as Julia sets have been intensively studied and the interested reader can consult the survey by Stallard [22. It is known that the Hausdorff dimension $H \operatorname{dim}\left(\mathcal{J}_{f}\right) \geq 1$ for every entire function $f$ since $\mathcal{J}_{f}$ contains non-degenerated continua [1]. Stallard [21] showed that $H \operatorname{dim}\left(\mathcal{J}_{f}\right)>1$ for all entire functions of class $\mathcal{B}$ which is the class of functions having a bounded set of singularities of the inverse. This result was later generalized by Rippon and Stallard to meromorphic functions of class $\mathcal{B}$ having finitely many poles [19] and by Bergweiler, Rippon, and Stallard to functions having a logarithmic tract over infinity [6].

The hyperbolic dimension of $f$, denoted HypDim $(f)$, is the supremum of the Hausdorff dimensions of all expanding repellers $X \subset \mathcal{J}_{f}$. Given this definition, $\operatorname{HypDim}(f) \leq H \operatorname{dim}\left(\mathcal{J}_{f}\right)$ but still we have the same kind of result since Barański, Karpińska, and Zdunik [2] have shown that $\operatorname{HypDim}(f)>1$ for every meromorphic function $f$ having a logarithmic tract over infinity. This result is of remarkable generality. Also it is sharp in the sense that $f_{\lambda}(z)=\lambda e^{z}, \lambda>0$ sufficiently small, has hyperbolic dimension arbitrarily close to one, a result due to Karpińska [12] (and Stallard 20] has shown that the Hausdorff dimension of the whole Julia set of entire functions can be arbitrarily close to one).

Nevertheless, we improve this result still for functions having a logarithmic tract $\Omega$ but with the additional assumption that one of these tracts has nice geometry, i.e., is a quasidisk, a John, or, more generally, a Hölder domain (see Definition 3.1).

Theorem 1.1. If a meromorphic map $f$ has a logarithmic tract over infinity, for example if $f \in \mathcal{B}$ and has a finite number of poles, and if this tract is Hölder, then

$$
\operatorname{Hyp} \operatorname{Dim}(f) \geq \Theta \geq 1 \text {. }
$$

Received by the editors September 13, 2017, and, in revised form, April 9, 2018.

2010 Mathematics Subject Classification. Primary 30D05, 37F10.

This research was supported in part by the Labex CEMPI (ANR-11-LABX-0007-01). 
If in addition the boundary of the tract has positive $\Theta$-length at every scale (see Definition 4.3), condition that is always satisfied if $\Theta=1$, then

$$
\operatorname{HypDim}(f)>\Theta \text {. }
$$

But what precisely is this number $\Theta$ ? Let us for the moment simply mention that we will provide examples showing that $\Theta$ can be any number in $[1,2[$. The precise definition of it is in Section 4.

In order to obtain a good lower estimate of the hyperbolic dimension we take into account the geometry of a logarithmic tract. It appears that the size of the Julia set and the hyperbolic dimension are influenced by the fractal behavior of the boundary of the tract near infinity. A logarithmic tract (over infinity) is a simply connected component $\Omega$ of $f^{-1}(\{|z|>R\})$, for some large $R$, such that $f_{\mid \Omega}: \Omega \rightarrow\{|z|>R\}$ is an infinite covering. The boundary $\partial \Omega$ is a smooth curve. Nevertheless, seen from infinity, $\partial \Omega$ can have fractal behavior. An appropriated way of measuring this is to employ integral means and $\beta$-numbers of natural rescalings of the Riemannian representation of the tract. This leads to a $\beta$-number at infinity, called $\beta_{\infty}$. Like in the classical case (see [16] and [13]), we will see that for Hölder tracts the function

$$
t \mapsto \beta_{\infty}(t)-t+1
$$

has a unique zero $\Theta$ and this turns out to be a better lower bound for the hyperbolic dimension.

We also investigate strict inequality between $\operatorname{Hyp} \operatorname{Dim}(f)$ and $\Theta$ since this has important applications in thermodynamic formalism (see [15]). Barański, Karpińska, and Zdunik do have strict inequality in their result in [2]. This implies that strict inequality holds if $\Theta=1$. In general, $\operatorname{Hyp} \operatorname{Dim}(f)=\Theta$ is perfectly possible. The strict inequality (1.2) only can hold if the tract has good additional geometric properties. We provide a condition (see Definition 4.3) imposing some growth of the integral means at the exponent $t=\Theta$ which then implies strict inequality (see Theorem 8.1).

For the hyperbolic dimension there exists a precise formula, called Bowen's Formula. A very general version of it is contained in [3]. Bowen's Formula involves the topological pressure and relies therefore on the whole dynamics. In the present paper, the equation $\beta_{\infty}(\Theta)-\Theta+1=0$ is a similar formula. It leads only to a minoration of the hyperbolic dimension. The advantage however is that $\beta_{\infty}$ does only depend on the function itself and not on the iterates. Consequently, the number $\Theta$ is often explicitly calculable.

Theorem 1.1 is formulated for global functions. What really matters is the behavior of the function $f$ in a logarithmic tract over infinity $\Omega$. So, we can consider in Theorem 1.1 local functions like $F=f_{\mid \Omega}$ as well. Keeping the point of view of the local functions, we provide examples for which the bound $\Theta$ is expressed in terms of the Minkowski dimension of a quasicircle and such that the range of this bound is any number in $[1,2[$.

\section{Preliminaries}

This note concerns transcendental meromorphic functions $f: \mathbb{C} \rightarrow \hat{\mathbb{C}}$ having a logarithmic tract over infinity. We denote $S(f)$ the singular set which is the closure of the critical and asymptotical values of $f$. The precise definition of the Julia set, which will be denoted by $\mathcal{J}_{f}$, and other dynamical features of transcendental functions are contained in the survey by Bergweiler [5]. We use standard notation 
such as $\mathbb{D}(z, r)=\{\mid z]<r\}, A(r, R)=\mathbb{D}(z, R) \backslash \overline{\mathbb{D}}(z, r)$, and $f \asymp g(f \preceq g)$ if $|f / g|$ is bounded below and above (bounded above) either by absolute constants or by constants where we indicate the dependence.

A key tool in dynamics is Koebe's Distortion Theorem. Theorem 1.3 in [16] is a version of it and from it follows that

$$
\frac{1}{8}(1-|z|) \leq \frac{\left|\psi^{\prime}(z)\right|}{\left|\psi^{\prime}(0)\right|} \leq \frac{2}{(1-|z|)^{3}} \quad \text { for every } z \in \mathbb{D}
$$

and for every univalent map $\psi$ defined on the unit disk $\mathbb{D}$ with values in $\mathbb{C}$.

An unbounded simply connected domain $\Omega \subset \mathbb{C}$ is a logarithmic tract over infinity of the function $f$ if there is $R>0$ such that $f_{\mid \Omega}: \Omega \rightarrow\{|z|>R\}$ is a universal covering. Then there exists a conformal map $\tau: \Omega \rightarrow\{\Re z>\log R\}$ such that (2.3) and $f=e^{\tau}$ on $\Omega$. Throughout the paper we will denote $\varphi=\tau^{-1}$ and suppose that $R=1$ so that $\varphi: \mathcal{H}=\{\Re z>0\} \rightarrow \Omega$. If $x>1$ and $z=x+i y \in \mathcal{H}$, then it follows from (2.1) by considering the univalent map $\psi$ defined by $\psi(\xi)=$ $\varphi(x \xi+x+i y), \xi \in \mathbb{D}$, that

$$
\frac{1}{8 x} \leq \frac{\left|\varphi^{\prime}(1+i y)\right|}{\left|\varphi^{\prime}(x+i y)\right|} \leq 2 x^{3} .
$$

Bishop's papers [7,8] give a complete new understanding of the functions of class $\mathcal{B}$. He shows that every local model of a tract $\tau: \Omega \rightarrow \mathcal{H}$ can be approximated by a global function $f \in \mathcal{B}$ and even in $\mathcal{S}$, the class of functions having only finitely many singularities of the inverse. His papers complete former work by Rempe-Gillen [18. Contrary to the next definition, Bishop considers much more general models where $\Omega$ can be an arbitrary union of simply connected unbounded domains.

Definition 2.1. A tract model $(\tau, \Omega)$ is a simply connected unbounded domain $\Omega$ along with a conformal map $\tau: \Omega \rightarrow \mathcal{H}$ such that

$$
\tau(z) \rightarrow \infty \text { if } z \rightarrow \infty .
$$

To every tract model $(\tau, \Omega)$ is associated a local, or model, function

$$
f=e^{\tau}: \Omega \rightarrow\{|z|>1\} .
$$

Its Julia set is $\mathcal{J}_{f}=\left\{z \in \Omega ; f^{n}(z) \in \Omega\right.$ for all $\left.n \geq 1\right\}$ and the hyperbolic dimension of a model function is defined precisely like the one of an entire function.

In the whole paper, $f$ is either a model function or a meromorphic function having a logarithmic tract. In both cases, $\Omega$ denotes a logarithmic tract and $\tau=$ $\varphi^{-1}: \Omega \rightarrow \mathcal{H}$ the conformal map such that $f_{\mid \Omega}=e^{\tau}$.

\section{HÖLDER TRACTS}

Let us now precisely define the notion of Hölder tracts. First of all, a simply connected hyperbolic domain $U \subset \mathbb{C}$ is Hölder if a conformal Riemann representation $h: \mathbb{D} \rightarrow U$ is Hölder: there exists $H>0$ and $0<\alpha \leq 1$ such that

$$
\left|h\left(z_{1}\right)-h\left(z_{2}\right)\right| \leq H\left|h^{\prime}(0)\right|\left|z_{1}-z_{2}\right|^{\alpha} \quad \text { for all } z_{1}, z_{2} \in \mathbb{D} .
$$

It is a well-known result by Hardy and Littlewood (see, for example, Theorem 5.1 in [10]) that (3.1) holds if and only if there exists $\hat{H}>0$ such that

$$
\left|h^{\prime}(z)\right| \leq \hat{H}\left|h^{\prime}(0)\right|\left(\frac{1}{1-|z|}\right)^{1-\alpha} \quad \text { for all } z \in \mathbb{D} .
$$


Moreover, the constants $\hat{H}$ and $H$ depend mutually on each other. To be more precise, (3.1) implies (3.2) with $\hat{H}=H$ and (3.2) implies (3.1) with $H=c \frac{\hat{H}}{\alpha}$ where $c$ is an absolute constant.

In both conditions (3.1) and (3.2) we have introduced the factor $\left|h^{\prime}(0)\right|$ in order to make them scale invariant. If the image domain $U$ is replaced by $\lambda U, \lambda \neq 0$, then these two conditions still hold with unchanged constants $H$ and $M$.

A Hölder domain is simply the image of the unit disk by a Hölder map. But such domains are clearly bounded whereas logarithmic tracts are unbounded domains. One might try to use spherical geometry but this is not really adapted here. A natural concept of Hölder tracts is the object of the following definition. It uses the notation

$$
Q_{T}=\{0<\Re z<2 T,-T<\Im z<T\}, \quad T>0,
$$

and a variant of (3.1): a conformal map $h: Q_{1} \rightarrow U$ is called $(H, \alpha)$-Hölder if

$$
\left|h\left(z_{1}\right)-h\left(z_{2}\right)\right| \leq H\left|h^{\prime}(1)\right|\left|z_{1}-z_{2}\right|^{\alpha} \quad \text { for all } \quad z_{1}, z_{2} \in Q_{1} .
$$

Definition 3.1. A tract model $(\Omega, \tau)$ is Hölder, more precisely $(H, \alpha)$-Hölder, if for every $\kappa \in(0,1)$ there exists $m>0$ and $T_{0} \geq 1$ such that, for every $T \geq T_{0}$,

(1) $\varphi_{T}=\tau^{-1} \circ T: Q_{1} \rightarrow \Omega_{T}=\tau^{-1}\left(Q_{T}\right)$ satisfies (3.3), and

(2) $\left|\varphi_{T}(z)\right| \geq m \operatorname{diam}\left(\Omega_{T}\right)$ for every $z \in Q_{1} \backslash Q_{\kappa}$.

Item (1) simply means that $\Omega$ is exhausted by a family of uniformly Hölder domains $\Omega_{T}$ and item (2) is a quantitative version of (2.3). Notice that it suffices to have item (2) for some $\kappa$, for example $\kappa=\frac{1}{2}$. Then one can easily recover the general case using (2.2) and the following lemma.

Lemma 3.2. If $h: Q_{1} \rightarrow U$ satisfies (3.3), then

$$
\left|h^{\prime}(1)\right| \asymp \operatorname{dist}(h(1), \partial U) \asymp \operatorname{diam}(U),
$$

where the involved constants depend only on $H$ and $\alpha$.

Proof. The relation $\left|h^{\prime}(1)\right| \preceq \operatorname{dist}(h(1), \partial U)$ results from Koebe's Distortion Theorem (see Corollary 1.4 in [16]). One always has $\operatorname{dist}(h(1), \partial U) \leq \operatorname{diam}(U)$ and (3.3) implies

Lemma 3.2 follows.

$$
\operatorname{diam}(U) \leq H\left|h^{\prime}(1)\right|(2 \sqrt{2})^{\alpha} .
$$

In the following, $T_{0}$ will be a sufficiently large number. It has to satisfy Definition 3.1 but also some other conditions. For example, we assume that

$$
|\varphi(T)| \geq 2|\varphi(0)| \text { for every } T \geq T_{0} .
$$

Then $|\varphi(T)| \asymp|\varphi(T)-\varphi(0)|$ and it follows from Lemma 3.2 that

$$
\left|\varphi^{\prime}(T) T\right|=\left|\varphi_{T}^{\prime}(1)\right| \asymp|\varphi(T)|, \quad T \geq T_{0} .
$$

If $\gamma$ is a rectifiable arc, then we denote $\gamma(a, b)$ a subarc of $\gamma$ joining $a, b \in \gamma$ and by $|\gamma(a, b)|$ its arc length. A simply connected domain $U \subset \mathbb{C}$ is called $c$-John with base point $x_{0} \in U, c>0$, if for every $x_{1} \in U$ there exists a rectifiable arc $\gamma \in U$ joining $x_{0}$ and $x_{1}$ such that

$$
\operatorname{dist}(x, \partial U) \geq c\left|\gamma\left(x, x_{1}\right)\right| \text { for every } \quad x \in U .
$$

John domains are Hölder but not vice versa (see [4] and [16]). We can therefore also consider the following class of John tracts. 
Definition 3.3. A tract model $(\Omega, \tau)$ is $c$-John if there exists $T_{0} \geq 1$ such that for every $T \geq T_{0}$ the domain $\Omega_{T}=\tau^{-1}\left(Q_{T}\right)$ is $c$-John with base point $z_{T}=\tau^{-1}(T)$.

A tract $\Omega$ of $f$ is a quasidisk and $\Gamma=\partial \Omega$ a quasicircle if the conformal map $\tau: \Omega \rightarrow \mathcal{H}$ has a quasiconformal extension to the plane. Quasidisks are John domains but the boundary of John domains is not necessarily a Jordan curve. Logarithmic tracts that are quasidisks are John tracts and thus Hölder tracts. So, logarithmic tracts that are quasidisks are examples of tracts that fit into our context. Here are more examples that the reader may have in mind.

Example 3.4. Let $P_{c}(z)=z^{2}+c$ be a polynomial with connected Julia set and having a repelling fixed point $p \in \partial A(\infty)$, the attracting basin of infinity of $P_{c}$. Then it is known that

(1) $A(\infty)$ is a John domain if and only if $P_{c}$ is semi-hyperbolic, a result by Carleson, Jones, and Yoccoz [9], and

(2) if $P_{c}$ is Collet-Eckmann, then $A(\infty)$ is a Hölder domain as Graczyk and Smirnov showed in [11.

There exists a linearizing entire function $f$ such that $f \circ \lambda=P_{c} \circ f$ where $\lambda$ is the multiplier of $P_{c}$ at $p$. If (1) or (2) holds, then $\Omega=f^{-1}(A(\infty))$ is, respectively, a John or Hölder tract (15] contains all the details and further information on that).

Here $\partial \Omega$ is a fractal and $\Omega$ is not exactly a tract as defined above. So, let $R>0$ sufficiently large such that $\{|z|=R\} \subset A(\infty)$. Then, the Julia set of $P_{c}$ being connected, $\{|z|>R\} \subset A(\infty)$ and $\Omega=f^{-1}(\{|z|>R\})$ is a tract as defined above. Here $\partial \Omega$ is smooth. But let us consider

$$
\partial \Omega \cap\left\{\left|\lambda^{n}\right| \leq|z|<\left|\lambda^{n+1}\right|\right\}, \quad n \geq 1 .
$$

If we rescale this set to unit size by multiplying it by $\lambda^{-n}$, then it follows from the linearizing functional equation that this set resembles more and more the fractal boundary of $A(\infty)$. In fact, these rescalings behave exactly like equipotential lines.

\section{INTEGRAL MEANS AT INFINITY}

Let us first have a look at the classical setting (see [13] and [16]) which concerns conformal maps on the unit disk. Let $h: \mathbb{D} \rightarrow W \subset \mathbb{C}$ be conformal and consider the classical $\beta$-function

$$
\beta_{h, \mathbb{D}}(t)=\limsup _{r \rightarrow 1} \frac{\log \int_{\{|\xi|=1\}}\left|h^{\prime}(r \xi)\right|^{t}|d \xi|}{\log \left(\frac{1}{1-r}\right)}
$$

measuring the growth of integral means of $\left|h^{\prime}\right|^{t}$ on circles of radius $0<r<1$. The behavior of this function is still not completely clear. If the range is a Hölder domain, then one knows (see [13, p. 241] as well as Corollary 10.18 in [16]) that

(a) there exists $0 \leq k<1$ such that $\beta_{h, \mathbb{D}}(t+s) \leq \beta_{h, \mathbb{D}}(t)+k s$ which implies that the convex function $t \mapsto b_{h, \mathbb{D}}(t)=\beta_{h, \mathbb{D}}(t)-t+1$ is strictly decreasing with $\lim _{t \rightarrow \infty} b_{h, \mathbb{D}}(t)=-\infty$.

(b) Since $b_{h, \mathbb{D}}(0)=1$, item (a) implies that there exists a unique $\Theta$ such that $\beta_{h, \mathbb{D}}(\Theta)=\Theta-1$.

Moreover, the number $\Theta$ is the Minkowski dimension of the boundary of the image domain.

Coming back to our setting, let $\varphi: \mathcal{H}=\{\Re z>0\} \rightarrow \Omega$ be a conformal map with $\varphi(\infty)=\infty$, i.e., such that $\lim _{z \rightarrow \infty}|\varphi(z)|=\infty$. In practice and up to normalization 
by an additive constant $\varphi$ will be the reciprocal of the conformal map $\tau$ used in the above definition of the logarithmic tract. Since we are interested in the fractal behavior of $\Omega$ near infinity it is natural to consider rescalings:

$$
g_{T}:=\frac{1}{\operatorname{diam} \Omega_{T}} \circ \varphi \circ T: Q_{1} \rightarrow \tilde{\Omega}_{T}=\frac{\Omega_{T}}{\operatorname{diam} \Omega_{T}}, T \geq 1 .
$$

If $\Omega$ is a Hölder tract, then $\left|g_{T}(z)\right| \geq m$ for every $z \in Q_{1} \backslash Q_{\kappa}$ and (3.3) holds with $\left|g_{T}^{\prime}(1)\right| \asymp 1$ and with constant independent of $T \geq T_{0}$. Let $z_{1}, z_{2} \in Q_{1} \backslash Q_{\kappa}$. Then $\left|g_{T}\left(z_{1}\right)-g_{T}\left(z_{2}\right)\right| \leq \tilde{H}$ with $\tilde{H}=\tilde{H}(H)$. Therefore,

$$
\frac{\left|\varphi\left(T z_{1}\right)\right|}{\left|\varphi\left(T z_{2}\right)\right|} \leq 1+\frac{\tilde{H}}{\left|g_{T}\left(z_{2}\right)\right|} \leq 1+\frac{\tilde{H}}{m}=: M \quad \text { for every } \quad z_{1}, z_{2} \in Q_{1} \backslash Q_{\kappa} .
$$

If the map $g_{T}$ is considered as a map on the segment $\{\Re z=1 / T\} \cap Q_{1}$ it is a rescaling of the map $\varphi$ defined on $\{\Re z=1\} \cap Q_{T}$. We now consider integral means of the rescaled maps. In order to do so, fix arbitrarily $\kappa \in(0,1)$, set

$$
\mathcal{E}=[-1,-\kappa] \cup[\kappa, 1] \quad \text { and } \quad \mathcal{E}_{T}=T \mathcal{E}=[-T,-\kappa T] \cup[\kappa T, T] .
$$

For $x \geq 1$ and $t \geq 0$ define

$$
I_{T}(x, t)=\int_{\mathcal{E}}\left|g_{T}^{\prime}\left(\frac{x}{T}+i y\right)\right|^{t} d y=\frac{T^{t-1}}{\left(\operatorname{diam} \Omega_{T}\right)^{t}} \int_{\mathcal{E}_{T}}\left|\varphi^{\prime}(x+i y)\right|^{t} d y .
$$

The integral means of scale $T$ will be

$$
\beta_{T}(t)=\frac{\log I_{T}(1, t)}{\log T}
$$

and the integral means function of the tract

$$
\beta_{\infty}(t)=\limsup _{T \rightarrow \infty} \beta_{T}(t), \quad t \geq 0 .
$$

In these definitions we used two arbitrary choices. First of all, any choice of the constant $\kappa \in(0,1)$ leads to the same function $\beta_{\infty}$. The second choice is that we considered the map $\varphi$ on the line $\{\Re z=1\}$ or, equivalently, we have taken $x=1$ in (4.5). Although we will use it differently, the following lemma explains that one could replace this line by any other line $\{\Re z=x\}$ without changing $\beta_{\infty}$.

Lemma 4.1. For every $x>1, t \geq 0, \kappa \in(0,1)$, and $T \geq 1$ we have that

$$
2^{-t} x^{-3 t} \leq \frac{\int_{\kappa T}^{T}\left|\varphi^{\prime}(x+i y)\right|^{t} d y}{\int_{\kappa T}^{T}\left|\varphi^{\prime}(1+i y)\right|^{t} d y} \leq 8^{t} x^{t}
$$

Proof. Follows directly from (2.2).

The function $\beta_{\infty}$ has exactly the same good properties as the standard $\beta$-function if $\Omega$ is a Hölder tract.

Proposition 4.2. Let $\Omega$ be a Hölder tract. Then items (a) and (b) of the classical setting mentioned above hold with $\beta_{h, \mathbb{D}}$ replaced by $\beta_{\infty}$ and there exists a unique number $\Theta$ such that

$$
\beta_{\infty}(\Theta)-\Theta+1=0
$$


Proof. This mainly follows from (3.2) by following the argument in [16]. Here is a way to adapt this proof to the present setting.

Let $T>1$ and let $g_{T}$ be the rescaled map defined in (4.2). We can consider $g$ as a map on the larger square $Q_{1+1 / T}$ and then it still is Hölder and we may assume that the constants $H, \alpha$ are unchanged. The segment $\{\Re z=1 / T\} \cap Q_{1}$ has distance $1 / T$ to the boundary of $Q_{1+1 / T}$. On the other hand, the Riemann map from $\mathbb{D}$ onto $Q_{1+1 / T}$ mapping 0 to 1 is, uniformly in $T>1$, bi-Hölder. It therefore follows from (3.2) that there exists $\tilde{M}>0$ such that

$$
\left|g_{T}^{\prime}(1 / T+i y)\right| \leq \tilde{M} T^{1-\alpha} \quad \text { for every }|y| \leq 1 \text { and every } T>1 .
$$

This inequality allows the following estimate: if $t, s>0$, then

$$
I_{T}(1, t+s)=\int_{\mathcal{E}}\left|g_{T}^{\prime}(1 / T+i y)\right|^{t+s} d y \leq \tilde{M}^{s} T^{(1-\alpha) s} I_{T}(1, t) .
$$

Consequently,

$$
\beta_{\infty}(t+s) \leq \beta_{\infty}(t)+(1-\alpha) s=\beta_{\infty}(t)+k s,
$$

where $k=1-\alpha \in[0,1$ [. The proof of the remaining affirmations of Proposition 4.2 is now straightforward.

The inequality (4.7) is formulated for $x=1$ and the analogous statement valid for any $x \geq 1$ is obtained in the same way. It implies that

$$
\lim _{T \rightarrow \infty} \frac{I_{T}(x, t)}{T^{t-1}}=0 \quad \text { if } \quad t>\Theta
$$

and

$$
\limsup _{T \rightarrow \infty} \frac{I_{T}(x, t)}{T^{t-1}}=\infty \quad \text { if } \quad t<\Theta .
$$

If $t=\Theta$, then $T^{1-t} I_{T}(x, t)$ can have arbitrary behavior as $T \rightarrow \infty$. The case when this quantity stays away from zero will be important later on.

Definition 4.3. The tract $\Omega$ has positive $t$-length at all scales if there exists $c>0$, $p \in[0,1[$ and $\varepsilon \in\{-,+\}$ such that

$$
\frac{I_{T}^{\varepsilon}(x, t)}{T^{t-1}}=\frac{1}{T^{t-1}} \int_{\varepsilon[\kappa, 1]}\left|g_{T}^{\prime}\left(\frac{x}{T}+i y\right)\right|^{t} d y \geq \frac{c}{x^{p}}
$$

for every $T \geq T_{0}$ and $1 \leq x \leq T$.

Clearly, we will use this notion for the critical value $t=\Theta$. We remark that it is natural to have a lower bound in (4.8) that tends to zero as $x \rightarrow \infty$. Indeed, $I_{T}(x, t)$ is related to the transfer operator as defined in [15. The behavior of this operator is completely described in [15] and it indicates that the natural choice for the exponent in (4.8) is $p=\Theta-1$.

The particular case of positive 1-length at all scales always holds. Notice that this implies the first step towards a proof of Theorem 1.1 since it gives the desired lower bound for $\Theta$.

Proposition 4.4. Every Hölder tract $\Omega$ has positive 1-length at all scales with $p=0$. Consequently, $\Theta \geq 1$. 
Proof. We show that (4.8) holds with $t=1$ and $p=0$. Notice that then

$$
\beta_{\infty}(1)=\limsup _{T \rightarrow \infty} \frac{\log I_{T}(1,1)}{\log T} \geq \lim _{T \rightarrow \infty} \frac{\log c}{\log T}=0,
$$

which implies that $\Theta \geq 1$.

Let $S$ be a hyperbolic geodesic of $Q_{1}$ joining $i b$ and $i$, where $\kappa \leq b<1$ is such that $S \subset Q_{1} \cap\{\Im z \geq \kappa\}$. Denote $z_{S} \in S$ a point realizing $r_{S}=\max \{\Re z, z \in S\}$. Let $S_{1}=S\left(i b, z_{S}\right)$ be the subarc of $S$ with endpoints $i b, z_{S}$ and let $S_{2}=S\left(z_{S}, i\right)$. We look for a lower bound of

$$
\int_{[\kappa, 1]}\left|g_{T}^{\prime}(x / T+i y)\right| d y \quad \text { where } T \geq 1 \text { and } x \geq 1 .
$$

Let $T \geq 1$. If $x / T>r_{S} / 2$, then it follows from Koebe's Distortion Theorem that $\left|g_{T}^{\prime}(x / T+i y)\right| \asymp\left|g_{T}^{\prime}(1)\right| \asymp 1$. It suffices thus to consider the case $0<\tilde{x}=x / T \leq$ $r_{S} / 2$. Then there are points $a_{i} \in S_{i}$ with $\Re a_{i}=\tilde{x}, i=1,2$. Let $S_{\tilde{x}}$ be the subarc of $S$ with endpoints $a_{1}, a_{2}$. Clearly, there exists a subarc $S_{0}$ of $S$ with distinct endpoints such that $S_{\tilde{x}} \supset S_{0}$ for all $0<\tilde{x} \leq r_{S} / 2$. Since all the arcs $S_{\tilde{x}}$ are hyperbolic segments, the Gehring-Hayman Theorem (see p. 72 in [16]) applies and shows that there exists an absolute constant $K$ such that

$$
\int_{[\kappa, 1]}\left|g_{T}^{\prime}\left(\frac{x}{T}+i y\right)\right| d y \geq\left|g_{T}\left(\left[a_{1}, a_{2}\right]\right)\right| \geq \frac{\left|g_{T}\left(S_{\tilde{x}}\right)\right|}{K} \geq \frac{\left|g_{T}\left(S_{0}\right)\right|}{K},
$$

where $|\sigma|$ stands for the euclidean length of an arc $\sigma$. This completes the proof because for the normalized functions $g_{T}$ we get, again from bounded distortion and Lemma 3.2. that $\inf _{T \geq 1}\left|g_{T}\left(S_{0}\right)\right|>0$.

\section{AN APPROPRIATED ITERATED FUNCTION SYSTEM}

By assumption, $f$ has a logarithmic Hölder tract $\Omega$. Let again $\varphi$ be the inverse of $\tau=\log f$ (modulo normalization by an additive constant) restricted to $\Omega$. We first need a technical lemma.

Lemma 5.1. There exists a constant $C>0$ such that

$$
|\varphi(a)| \leq C \Im(a)^{2}
$$

for all $a \in \mathcal{H}$ with $\max \{1, \Re(a)\} \leq 2|\Im(a)|$.

Proof. Let $T \geq T_{0}$ and remember that $\varphi_{T}=\varphi \circ T: Q_{1} \rightarrow \Omega_{T}$ satisfies the Hölder inequality (3.3). Since $\left|\varphi_{T}^{\prime}(1)\right|=T\left|\varphi^{\prime}(T)\right|$ and since (2.2) implies $\left|\varphi^{\prime}(T)\right| \leq$ $8\left|\varphi^{\prime}(1)\right| T$, it follows from (3.3) that

$$
\left|\varphi\left(z_{1}\right)-\varphi\left(z_{2}\right)\right| \leq 8 H\left|\varphi^{\prime}(1)\right| T^{2-\alpha}\left|z_{1}-z_{2}\right|^{\alpha}, \quad z_{1}, z_{2} \in Q_{T} .
$$

Consider now a point $a \in \mathcal{H}$ and set $T=\max \left\{\frac{1}{2} \Re(a),|\Im(a)|\right\}$. Then $a \in \bar{Q}_{T}$ and it follows from (5.2) with $z_{1}=0$ and $z_{2}=a$ that

$$
|\varphi(a)-\varphi(0)| \preceq T^{2-\alpha}|a|^{\alpha} .
$$

The inequality (5.1) now follows easily.

Increasing $T_{0}$ if necessary we may assume that it satisfies

$$
T_{0} \geq \max \{C, e\} \quad \text { and } \min _{|T| \geq T_{0}}|\varphi(1+i T)| \geq e,
$$

where $C$ is the constant from Lemma 5.1, Then we have the following observation which is similar to Corollary 3 in [2]. 
Lemma 5.2. For every $|T| \geq T_{0}$ there exists $1 \leq x \leq 3 \log |T|$ such that

$$
\left|f\left(b_{T}\right)\right|=\left|b_{T}\right| \text { where } b_{T}=\varphi(x+i T) .
$$

Proof. Since $\left|f\left(b_{T}\right)\right|=e^{x}$ we have to show that for every $T \geq T_{0}$ there exists $1 \leq x \leq 3 \log T$ such that $e^{x}=|\varphi(x+i T)|$. But this results from (5.3) combined with (5.1).

In order to get the required iterated function system, we look for domains $V_{k}, V$ such that $\bar{V}_{k} \subset V$ and such that some inverse branch of $f$ maps conformally $V$ onto $V_{k}$ where $1 \leq k \leq N$ and where the number of generators $N$ is as large as possible.

In the following we use only positive values of $T$ in Lemma 5.2. The construction of the iterated function system is totally symmetric and so one can take instead throughout the whole construction negative values of $T$.

Let $T_{1} \geq T_{0}$ and let $N \geq 1$. Lemma 5.2 shows that there exists a point $a_{1}=$ $x+i T_{1}$ with $1 \leq x \leq 3 \log T_{1}$ such that $\left|f\left(b_{1}\right)\right|=\left|b_{1}\right|$ where $b_{1}=\varphi\left(a_{1}\right)$. For $1 \leq k \leq N$ let $a_{k}=a_{1}+2 i \pi(k-1)$ and let $b_{k}=\varphi\left(a_{k}\right)$. Define further

$$
R_{1}=\min _{1 \leq k \leq N}\left|b_{k}\right| \leq \max _{1 \leq k \leq N}\left|b_{k}\right|=R_{2} .
$$

Then, all the points $b_{k}$ are in the annulus $A\left(R_{1}, R_{2}\right)=\left\{R_{1} \leq|z| \leq R_{2}\right\}$ and they have the same image $\tilde{b}=f\left(b_{k}\right)$. Set

$$
T_{k}=\Im\left(a_{k}\right), \quad 1 \leq k \leq N .
$$

Inequality (5.1) of Lemma 5.1 implies that

$$
\left|b_{k}\right| \leq C T_{k}^{2} \text { for all } 1 \leq k \leq N .
$$

In particular, $R_{1} \leq C T_{1}^{2}$ and $R_{2} \leq C T_{N}^{2}$.

Let $M$ be the constant from (4.3). Consider the annulus

$$
A=A\left(\frac{R_{1}}{M}, M R_{2}\right) \quad \text { and let } \quad V=A \backslash \gamma,
$$

where $\gamma$ is a connected component of $\varphi(] 0, \infty[) \cap A$ such that $\gamma$ is a crosscut of $A$ with one endpoint in each boundary component of $A$. We may assume that $\tilde{b} \notin \gamma$ since otherwise it suffices to replace $\gamma$ by another arc that is arbitrarily close to it. Let finally $U_{k}$ be the connected component of $\exp ^{-1}(V)$ that contains $a_{k}$ and $V_{k}=\varphi\left(U_{k}\right), 1 \leq k \leq N$.

Up to now, there is no restriction in this construction except the lower bound $T_{1} \geq T_{0}$. In the applications later on we can take $T_{1}$ arbitrarily large but we need a large number $N$ of generators of the iterated function system. We now estimate how large this number can be.

Lemma 5.3. There exists $\varepsilon>0$ and $T_{0}$ such that the following holds:

$$
\text { if } T_{1} \geq T_{0} \text { and } N \geq 1 \text { are such that } \varepsilon T_{1}>\log T_{N} \text {, }
$$

then $\bar{V}_{k} \subset V$ for all $1 \leq k \leq N$.

Proof. We first establish two estimates. We know from (5.5) that $R_{2} \leq C T_{N}^{2}$. Condition (5.6) therefore implies

$$
\log \left(M R_{2}\right) \leq \log (M C)+2 \log T_{N}<\log (M C)+2 \varepsilon T_{1}
$$

and also

$$
\log \left(M^{2} \frac{R_{2}}{R_{1}}\right) \leq \log \left(M^{2} R_{2}\right)<\log \left(M^{2} C\right)+2 \varepsilon T_{1} .
$$


Claim 5.4. There exists $B \geq 1$ such that

$$
\bar{U}_{k} \subset\left\{T_{k}-\delta<\Im \xi<T_{k}+\delta\right\}, \quad \text { where } \delta=B \log \left(M^{2} \frac{R_{2}}{R_{1}}\right) .
$$

Let us admit this claim for a moment. We have

$$
\kappa\left(T_{k}+\delta\right)<T_{k}-\delta \text { for all } 1 \leq k \leq N
$$

if $(1+\kappa) \delta<(1-\kappa) T_{1}$. Inequality (5.8) shows that this is the case if

$$
\frac{1+\kappa}{1-\kappa} B \log \left(M^{2} C\right)+2 \varepsilon B \frac{1+\kappa}{1-\kappa} T_{1}<T_{1} .
$$

This is true if we choose $\varepsilon=(1-\kappa) /(4 B(1+\kappa))$ and if we fix $T_{0}$ large enough. Notice that it then follows from (5.7) that

$$
\log \left(M R_{2}\right)<T_{1}
$$

which itself implies

$$
\bar{U}_{k} \subset\left\{\log \frac{R_{1}}{M} \leq \Re \xi \leq \log \left(M R_{2}\right)\right\} \subset\left\{0<\Re \xi<2\left(T_{k}+\delta\right)\right\}, \quad 1 \leq k \leq N .
$$

On the other hand, Claim 5.4 and (5.9) imply

$$
\bar{U}_{k} \subset\left\{\kappa\left(T_{k}+\delta\right)<\Im \xi<T_{k}+\delta\right\}
$$

and thus

$$
\bar{U}_{k} \subset \Delta_{k}:=Q_{T_{k}+\delta} \backslash \bar{Q}_{\kappa\left(T_{k}+\delta\right)}, \quad 1 \leq k \leq N .
$$

Since $a_{k} \in U_{k} \subset \Delta_{k}$ it suffices now to use (4.3) in order to get

$$
\bar{V}_{k}=\varphi\left(\bar{U}_{k}\right) \subset A\left(\frac{\left|b_{k}\right|}{M}, M\left|b_{k}\right|\right) \subset A\left(\frac{R_{1}}{M}, M R_{2}\right)=A, \quad 1 \leq k \leq N .
$$

By construction $\bar{V}_{k} \cap \gamma=\emptyset$ and thus $\bar{V}_{k} \subset V$ for all $1 \leq k \leq N$.

It remains to show Claim 5.4. Let $0<t_{0}<t_{1}<\ldots<t_{\nu}$, set $w_{j}=\varphi\left(t_{j}\right)$, and assume that $w_{j} \in \gamma$. Fix arbitrarily $k \in\{1, \ldots, N\}$ and let $\sigma_{k} \subset \partial U_{k}$ be an arc such that $\gamma=e^{\sigma_{k}}$. Let finally $\xi_{j} \in \sigma_{k}$ be such that $e^{\xi_{j}}=w_{j}$ and assume that

$$
\left|\Im \xi_{j+1}-\Im \xi_{j}\right|=2 \pi, \quad 0 \leq j \leq \nu .
$$

This last relation implies that there exists $\left.s_{j} \in\right] t_{j}, t_{j+1}[$ such that

$$
\operatorname{Arg}\left(\varphi\left(s_{j}\right)\right)+\pi=\operatorname{Arg}\left(w_{j}\right)=\operatorname{Arg}\left(w_{j+1}\right) \bmod 2 \pi .
$$

Since $T_{k}=\Im\left(a_{k}\right)$ the affirmation of the claim follows if we have an appropriate estimate of $\max \left\{\left|\Im\left(\xi-a_{k}\right)\right| ; \quad \xi \in U_{k}\right\}$. This maximum can be estimated by the maximal number $\nu$ of points $\xi_{j} \in \sigma_{k}$ that satisfy (5.12). We now estimate $\nu$. Observe first that $\left|\varphi^{\prime}\left(t_{j}\right)\right| t_{j} \asymp\left|w_{j}\right|, 0 \leq j \leq \nu$ (see (3.4) ). It therefore results from Koebe's Distortion Theorem that there exists $0<k_{*}<1$ such that

$$
\begin{gathered}
\varphi\left(\mathbb{D}\left(t_{j}, \frac{t_{j}}{2}\right)\right) \supset \mathbb{D}\left(w_{j}, k_{*}\left|w_{j}\right|\right) \quad \text { and } \\
\varphi^{-1}\left(\mathbb{D}\left(w_{j}, k_{*}\left|w_{j}\right|\right)\right) \supset \mathbb{D}\left(t_{j}, k_{*}^{2} t_{j}\right), \quad j=0, \ldots, \nu .
\end{gathered}
$$


The origin is not in the disks $\mathbb{D}\left(w_{j}, k_{*}\left|w_{j}\right|\right)$. It thus follows from (5.13) that $\varphi\left(s_{i}\right) \notin \mathbb{D}\left(w_{j}, k_{*}\left|w_{j}\right|\right)$ and thus from (5.15)

$$
s_{i} \notin \mathbb{D}\left(t_{j}, k_{*}^{2} t_{j}\right) \quad \text { for all } \quad 0 \leq i<\nu, 0 \leq j \leq \nu .
$$

Consequently, the disks $\mathbb{D}\left(t_{j}, k_{*}^{2} t_{j}\right)$ are pairwise disjoint. Like in (15.14), there exists $k>0$ such that $\varphi\left(\mathbb{D}\left(t_{j}, k_{*}^{2} t_{j}\right)\right) \supset \mathbb{D}\left(w_{j}, k\left|w_{j}\right|\right)$ and clearly the latter disks are also pairwise disjoint. Therefore, and since the points $w_{j}$ are on a ray emanating from the origin, if $\left|w_{i}\right|<\left|w_{j}\right|$, then

$$
(1+k)\left|w_{i}\right|<\left|w_{j}\right| .
$$

Since $R_{1} / M \leq\left|w_{i}\right| \leq M R_{2}$ it follows that $(1+k)^{\nu} \frac{R_{1}}{M} \leq M R_{2}$ which itself implies

$$
\nu \preceq \log \left(M^{2} \frac{R_{2}}{R_{1}}\right) .
$$

Claim 5.4 is now a straightforward consequence of this estimate.

The map $f: V_{k} \rightarrow V$ is conformal. Denote $\Phi_{k}: V \rightarrow V_{k}$ the inverse map. We now clearly have the following statement.

Proposition 5.5. There exists $\varepsilon>0$ and $T_{0}$ such that the following holds. For every $T_{1} \geq T_{0}$ and $N \geq 1$ such that (5.6) is satisfied the maps

$$
\Phi_{k}: V \rightarrow V_{k}, \quad 1 \leq k \leq N,
$$

define a (finite) conformal iterated function system as defined in [14] with limit set a subset of the Julia set of $f$.

\section{Minoration of the hyperbolic dimension by $\Theta$}

One main goal of this note is to establish the following result. Here we associate to a logarithmic tract the function $\beta_{\infty}$ as explained in Section 4 ,

Theorem 6.1. Let $f: \mathbb{C} \rightarrow \hat{\mathbb{C}}$ be a model or a meromorphic function having a logarithmic tract $\Omega$. Assume that $\Omega$ is Hölder and let $\Theta$ be the unique zero of the function $t \mapsto \beta_{\infty}(t)-t+1$. Then,

$$
\operatorname{HypDim}(f) \geq \Theta \geq 1 \text {. }
$$

Proof of Theorem 6.1. The proof uses only the behavior of $f$ in the tract. So it does not matter whether $f$ is a global function or a model. Also, we already know that $\Theta \geq 1$ (Proposition 4.4). It remains to compare the hyperbolic dimension of $f$ and $\Theta$.

In order to prove the required inequality we keep the notation of Section 5 and take the iterated function system from Proposition 5.5 with number of mappings $N \geq 1$ maximal such that

$$
\Im a_{N} \leq \kappa^{-1} T_{1}=: T .
$$

Notice that the condition (5.6) clearly holds provided we take $T_{1} \geq T_{0}$ sufficiently large (see (5.5)). 
We recall that in the construction of the iterated function system positive values of $T_{1}$ have been used. For the present proof this is possible if

$$
\int_{\kappa}^{1}\left|g_{T_{1}}^{\prime}\left(1 / T_{1}+i y\right)\right|^{t} d t \geq \frac{1}{2} \int_{\mathcal{E}}\left|g_{T_{1}}^{\prime}\left(1 / T_{1}+i y\right)\right|^{t} d t=\frac{1}{2} I_{T_{1}}(1, t) .
$$

Without loss of generality we may suppose that this is the case.

We must estimate the Hausdorff dimension of the limit set of this iterated function system. In order to do so we will work with the cylindrical metric $|d z| /|z|$. The derivative $\left|f^{\prime}(z)\right|_{1}$ of $f$ at a point $z \in \Omega$ with respect to this metric is

$$
\left|f^{\prime}(z)\right|_{1}=\frac{\left|f^{\prime}(z)\right|}{|f(z)|}|z|=\frac{\left|e^{\tau(z)} \tau^{\prime}(z)\right|}{\mid e^{\tau(z) \mid}}|z|=\frac{|\varphi(w)|}{\left|\varphi^{\prime}(w)\right|} \text { with } w=\tau(z) .
$$

Let $t>0$ and let $w \in V$. We have to estimate $\Sigma_{t}(w)=\sum_{k=1}^{N}\left|\Phi_{k}^{\prime}(w)\right|_{1}^{t}$. Fix $w \in V$ and let $\xi_{k} \in U_{k}$ such that $\exp \left(\xi_{k}\right)=w$. Then $\xi_{k}=\xi_{1}+2 \pi i(k-1)$ and

$$
\Sigma_{t}(w)=\sum_{k=1}^{N}\left|\Phi_{k}^{\prime}(w)\right|_{1}^{t}=\sum_{k=1}^{N}\left|\frac{\varphi^{\prime}\left(\xi_{k}\right)}{\varphi\left(\xi_{k}\right)}\right|^{t} .
$$

By Koebe's Distortion Theorem $\left|\varphi^{\prime}\left(\xi_{k}\right)\right| \asymp\left|\varphi^{\prime}\left(\xi_{k}+i y\right)\right|$ for $0 \leq y \leq 2 \pi$. Item (2) of Definition 3.1 and $\xi_{k} \in Q_{T} \backslash Q_{\kappa T}$ imply $\left|\varphi\left(\xi_{k}\right)\right| \asymp \operatorname{diam}\left(\Omega_{T}\right)$. Consequently,

$$
\Sigma_{t}(w) \asymp \frac{1}{\operatorname{diam}\left(\Omega_{T}\right)^{t}} \int_{\kappa T}^{T}\left|\varphi^{\prime}(x+i y)\right|^{t} d y,
$$

where $x=\log R_{1} \in\left[1,3 \log T_{1}\right]$. Lemma 4.1 yields

$$
\frac{1}{\left(2 x^{3}\right)^{t}} \int_{\kappa T}^{T}\left|\varphi^{\prime}(1+i y)\right|^{t} d y \leq \int_{\kappa T}^{T}\left|\varphi^{\prime}(x+i y)\right|^{t} d y .
$$

So, let us consider

$$
\frac{1}{\operatorname{diam}\left(\Omega_{T}\right)^{t}} \int_{\kappa T}^{T}\left|\varphi^{\prime}(1+i y)\right|^{t} d y=T^{1-t} \int_{\kappa}^{1}\left|g_{T}^{\prime}\left(\frac{1}{T}+i y\right)\right|^{t} d y,
$$

where the rescaled function $g_{T}$ has been defined in (4.2). We have (6.1) and thus

$$
\frac{1}{\operatorname{diam}\left(\Omega_{T}\right)^{t}} \int_{\kappa T}^{T}\left|\varphi^{\prime}(1+i y)\right|^{t} d y \geq \frac{1}{2} T^{1-t} I_{T}(1, t)=\frac{1}{2} T^{1-t+\beta_{T}(t)}
$$

from which follows that

$$
\frac{1}{(\log T)^{3 t}} T^{1-t+\beta_{T}(t)} \preceq \frac{1}{x^{3 t}} T^{1-t+\beta_{T}(t)} \preceq \Sigma_{t}(w) .
$$

Let $t<\Theta$. Then there exists $\eta>0$ and $T>1$ arbitrarily large such that

$$
\beta_{T}(t)-t+1 \geq \eta>0 .
$$

Combined with (6.3) this shows that there exists $R$ and thus $T$ sufficiently large such that

$$
\Sigma_{t}(w) \geq 2 \quad \text { for every } \quad w \in V .
$$

This implies that for every $t<\Theta$ the iterated function system has positive pressure and thus the Hausdorff dimension of the limit set of this system is strictly larger than $t$ (see [14] or [17]). We therefore proved that HypDim $(f) \geq \Theta$. 


\section{EXAMPLES WITH FRACTAL TRACT}

7.1. Linearizers. Classical functions like $\lambda e^{z^{d}}$ have logarithmic tracts with $\Theta=$ 1. The functions of Example 3.4 are typical examples of entire functions having fractal Hölder tracts, i.e., their tracts have $\Theta>1$. More precisely, for every entire linearizing function $f$ of a polynomial $P$ having connected Julia set we have the following (see Theorem 7.8 in [15]):

(1) The logarithmic tracts of $f$ are Hölder if and only if $P$ is topological ColletEckmann.

(2) The $\Theta$-number of the tracts of $f$ is precisely the hyperbolic dimension of the polynomial $P$. Moreover, $\Theta>1$ except if $P(z)=c z^{d}, d \geq 2$, or if $P$ is a Tchebychev polynomial (this relies on [23]).

Consequently, the number $\Theta$ admits all values in $[1,2$ [ already for this particular class of entire functions.

7.2. Models with fractal quasicircle tract. Here we present a family of models that have a fractal tract. For these examples the number $\Theta$ is precisely known. It is exactly given by the Minkowski dimension of a generating quasicircle.

Let $\sigma$ be a bounded quasicircle such that $\sigma \cap[0, \infty[=\{1\}$ and such that the origin is in the bounded component $U$ of $\mathbb{C} \backslash \sigma$. Let $h: \mathbb{D} \rightarrow U$ ba any conformal map and let $\beta_{h, \mathbb{D}}$ be the $\beta$-function of the interior of this curve as defined in (4.1). Consider then $\gamma_{1}$ the lift by the exponential map of $\sigma$ having endpoints $2 i \pi$ and $4 i \pi$. Define $\gamma_{k}=2^{k-1} \gamma_{1}, k \in \mathbb{Z}$, and

$$
\Gamma^{+}=\bigcup_{k \in \mathbb{Z}} \gamma_{k} \quad \text { and } \quad \Gamma=-\Gamma^{+} \cup\{0\} \cup \Gamma^{+} .
$$

This curve $\Gamma$ is a quasicircle. If $\Omega$ is one of the connected components of $\mathbb{C} \backslash \Gamma$ and $\tau: \Omega \rightarrow \mathcal{H}$ a conformal map, then $(\tau, \Omega)$ is a tract model. Let $f=\exp ^{\tau}$ be the associated model function and let $\Theta$ be the number associated to the quasidisk, thus Hölder, tract $\Omega$.

By construction of $\Gamma$, a simple verification shows that the $\beta_{\infty}$-function of $(\tau, \Omega)$ is equal to $\beta_{h, \mathbb{D}}$. Theorem 6.1 and Corollary 10.18 in 16 imply now

$$
\operatorname{HypDim}(f) \geq \Theta=\operatorname{Mdim}(\sigma) \text {. }
$$

These examples can be easily generalized in many ways simply by taking for every $k \in \mathbb{Z}$ a different generating curve $\sigma_{k}$ in order to define $\gamma_{k}$.

\section{StRict INEQUALity}

In general, one cannot expect to have strict inequality between $\Theta$ and the hyperbolic dimension. There must be an additional assumption in order to expect strict inequality in (7.1). This is the point where the positive $\Theta$-length on all scale conditions comes into play.

Theorem 8.1. Let $f: \mathbb{C} \rightarrow \hat{\mathbb{C}}$ be a model or meromorphic function having a logarithmic tract $\Omega$. Assume that $\Omega$ is Hölder and let $\Theta$ be the unique zero of the function $t \mapsto \beta_{\infty}(t)-t+1$. If $\Omega$ has positive $\Theta$-length at all scales, then

$$
\operatorname{HypDim}(f)>\Theta \text {. }
$$


Let us first illustrate how this result can be used and then provide a proof. Recall from Proposition 4.4 that the assumption of Theorem 8.1 always holds for free if $\Theta=1$. Combined with Theorem 6.1 this implies the following.

Corollary 8.2. If $f$ has a logarithmic Hölder tract, then HypDim $(f)>1$.

But this is only a new point of view of the result by Barański, Karpińska, and Zdunik [2] done under the additional Hölder assumption.

Coming back to the models of Section 7.2 the $\Theta$-length assumption on all scales is easy to check if the generating curve has positive $\Theta$-measure.

Proposition 8.3. Suppose $f$ is as in Section 7.2 , that $\Theta=\operatorname{Mdim}(\sigma)<2$, and that $\sigma$ has $\Theta$-Hausdorff measure $H M^{\Theta}(\sigma)>0$. Then

$$
\operatorname{Hyp} \operatorname{Dim}(f)>\Theta \text {. }
$$

Proof. We have to check that the tract of $f$ has positive $\Theta$-length at all scales since then the result follows from Theorem 8.1

The domain $\Omega$ has the invariance $2 \Omega=\frac{1}{2} \Omega=\Omega$ and the points $b_{k}=2^{k} i \pi, k \in \mathbb{Z}$, are in the boundary $\Gamma$. Let $a_{k}=\varphi^{-1}\left(b_{k}\right), k \in \mathbb{Z}$, and $\mu=a_{2} / a_{1}$. This invariance implies that the conformal map $\varphi_{1}=\frac{1}{2} \varphi \circ \mu$ also maps $\mathcal{H}$ onto $\Omega$ and thus $\varphi^{-1} \circ \varphi_{1}$ is a conformal map of $\mathcal{H}$ that fixes the three points $0, a_{1}$, and $\infty$. Consequently, $\varphi_{1}=\varphi$ and it follows inductively that

$$
\frac{1}{2^{n}} \varphi \mu^{n}=\varphi \text { and } a_{n}=\mu^{n-1} a_{1} \text { for every } n \geq 1 .
$$

We may assume that $a_{1}=i$ so that $a_{n}=\mu^{n-1} i$.

In order to verify (4.8) with $t=\Theta$ we may assume that $\kappa=\mu^{-1}$ and take $T=\mu^{n}, n \geq 1$. Let $1 \leq x \leq T$ and $s=\mu^{-n} x$. Since (8.1) signifies that the rescaled map $g_{\mu^{n}}=\varphi$ we have

$$
I_{T}^{+}(x, \Theta)=\int_{\kappa}^{1}\left|\varphi^{\prime}\left(\mu^{-n} x+i y\right)\right|^{\Theta} d y \asymp s^{1-\Theta} \sum_{k}\left(\left|\varphi^{\prime}(s+i s k)\right| s\right)^{\Theta},
$$

where the sum is taken over all integers $k$ such that $\kappa \leq s k<1$. The curve $\Gamma$ being a quasicircle, the latter sum can be associated with $\sum_{k} \operatorname{diam}\left(D_{k}\right)^{\Theta}$ where the $D_{k}$ are disks that cover $\gamma_{0}$ and have

$$
\operatorname{diam}\left(D_{k}\right) \asymp\left|\varphi^{\prime}(s+i s k)\right| s .
$$

By assumption, $H M^{\Theta}\left(\gamma_{0}\right)=m>0$ which implies that there exists $r_{0}>0$ such that $\sum_{k} \operatorname{diam}\left(D_{k}\right)^{\Theta} \geq m / 2$ provided $\operatorname{diam}\left(D_{k}\right) \leq r_{0}$ for all $k$. Uniform continuity of $\varphi$ in a compact neighborhood in $\overline{\mathcal{H}}$ of $\mathcal{E}$ allows one to choose $s_{0}>0$ such that the disks $D_{k}$ above have $\operatorname{diam}\left(D_{k}\right)<r_{0}$ if $s<s_{0}$. Therefore,

$$
\frac{I_{T}^{+}(x, \Theta)}{T^{\Theta-1}} \asymp \mu^{n(1-\Theta)} s^{1-\Theta} \sum_{k} \operatorname{diam}\left(D_{k}\right)^{\Theta} \geq \frac{m}{2} x^{1-\Theta}
$$

provided $s=\mu^{-n} x<s_{0}$. This shows (4.8) with $\varepsilon=+$ and $p=\Theta-1$ for all $1 \leq x<\mu^{n} s_{0}=T s_{0}$.

It remains to consider $x \in[1, T]$ such that $x \geq T s_{0}$. But then

$$
\left|\varphi^{\prime}(s+i y)\right|=\left|\varphi^{\prime}\left(\frac{x}{R}+i y\right)\right| \asymp\left|\varphi^{\prime}(1)\right| \text { for all } y \in \mathcal{E}
$$

by bounded distortion from which (4.8) with $p=\Theta-1 \in[0,1[$ also follows. 
Proof of Theorem 8.1. By assumption, $\Omega$ has positive $\Theta$-length at all scales and we can assume that $\varepsilon=+$. We use again the iterated function system and the notation of Section 5. Let $T_{1} \geq T_{0}$. Choose the number $N$ of mappings of the iterated function system such that $T_{N} \asymp T_{1}^{2}$. Again, the condition (5.6) is clearly satisfied provided we take $T_{1}$ sufficiently large. Let $m$ be the maximal integer such that $T_{N} \kappa^{m} \geq T_{1}$. Then $m \asymp \log T_{1}$.

Let $t_{n}=\kappa^{n} T_{N}$. If $w \in V \subset A\left(\frac{R_{1}}{M}, M R_{2}\right)$, then

$$
x=\log |w| \leq \log \left(M R_{2}\right)<T_{1} \leq t_{n}
$$

by (5.10). We can therefore apply the assumption that $\Omega$ has positive $\Theta$-length at all scales with $x=\log |w|$ and $T=t_{n}$ :

$$
t_{n}^{1-\Theta} I_{t_{n}}^{+}(\log |w|, \Theta) \geq \frac{c}{(\log |w|)^{p}} \quad \text { for all } \quad w \in V \text { and } 0 \leq n \leq m .
$$

Proceed now exactly like in the proof of Theorem 6.1 up to (6.2):

$$
\Sigma_{\Theta}(w)=\sum_{k=1}^{N}\left|\left(\Phi_{k}\right)^{\prime}(w)\right|_{1}^{\Theta}=\sum_{k=1}^{N}\left|\frac{\varphi^{\prime}\left(\xi_{k}\right)}{\varphi^{\left(\xi_{k}\right)}}\right|^{\Theta} \geq \sum_{n=0}^{m-1} \sum_{t_{n+1} \leq \Im \xi_{k}<t_{n}}\left|\frac{\varphi^{\prime}\left(\xi_{k}\right)}{\varphi\left(\xi_{k}\right)}\right|^{\Theta} .
$$

The last sum can again be replaced by an integral so that

$$
\Sigma_{\Theta}(w) \succeq \sum_{n=0}^{m-1}\left(\operatorname{diam} \Omega_{t_{n}}\right)^{-\Theta} \int_{t_{n+1}}^{t_{n}}\left|\varphi^{\prime}(\log |w|+i y)\right|^{\Theta} d y \geq m \frac{c}{(\log |w|)^{p}}
$$

by (8.3). From (5.5) it follows that $|w| \preceq R_{2} \preceq T_{N}^{2} \asymp T_{1}^{4}$. Since $m \asymp \log T_{1}$ we finally get $\Sigma_{\Theta}(w) \succeq\left(\log T_{1}\right)^{1-p}$. It suffices now to choose $T_{1} \geq T_{0}$ sufficiently large such that

$$
\Sigma_{\Theta}(w) \geq 2 \text { for every } \quad w \in V .
$$

This implies that the topological pressure at $t=\Theta$ of the iterated function system is strictly positive. Again by [14] or [17] it follows finally that the Hausdorff dimension of the limit set is strictly larger than $\Theta$.

Proof of Theorem 1.1. This is now a direct consequence of Proposition 4.4, Theorem 6.1, and Theorem 8.1.

\section{REFERENCES}

[1] I. N. Baker, The domains of normality of an entire function, Ann. Acad. Sci. Fenn. Ser. A I Math. 1 (1975), no. 2, 277-283. MR0402044 t62

[2] Krzysztof Barański, Bogusława Karpińska, and Anna Zdunik, Hyperbolic dimension of Julia sets of meromorphic maps with logarithmic tracts, Int. Math. Res. Not. IMRN 4 (2009), 615-624, DOI 10.1093/imrn/rnn141. MR2480096 恀, 63 69, 75

[3] Krzysztof Barański, Bogusława Karpińska, and Anna Zdunik, Bowen's formula for meromorphic functions, Ergodic Theory Dynam. Systems 32 (2012), no. 4, 1165-1189, DOI 10.1017/S0143385711000290. MR2955309 163

[4] Jochen Becker and Christian Pommerenke, Hölder continuity of conformal mappings and nonquasiconformal Jordan curves, Comment. Math. Helv. 57 (1982), no. 2, 221-225, DOI 10.1007/BF02565858. MR684114 65

[5] Walter Bergweiler, Iteration of meromorphic functions, Bull. Amer. Math. Soc. (N.S.) 29 (1993), no. 2, 151-188, DOI 10.1090/S0273-0979-1993-00432-4. MR1216719 63

[6] Walter Bergweiler, Philip J. Rippon, and Gwyneth M. Stallard, Dynamics of meromorphic functions with direct or logarithmic singularities, Proc. Lond. Math. Soc. (3) 97 (2008), no. 2, 368-400, DOI 10.1112/plms/pdn007. MR2439666 t62

[7] Christopher J. Bishop, Models for the Eremenko-Lyubich class, J. Lond. Math. Soc. (2) 92 (2015), no. 1, 202-221, DOI 10.1112/jlms/jdv021. MR3384512 64 
[8] Christopher J. Bishop, Models for the Speiser class, Proc. Lond. Math. Soc. (3) 114 (2017), no. 5, 765-797, DOI 10.1112/plms.12025. MR3653246 64

[9] Lennart Carleson, Peter W. Jones, and Jean-Christophe Yoccoz, Julia and John, Bol. Soc. Brasil. Mat. (N.S.) 25 (1994), no. 1, 1-30, DOI 10.1007/BF01232933. MR1274760 66

[10] Peter L. Duren, Theory of $H^{p}$ spaces, Pure and Applied Mathematics, Vol. 38, Academic Press, New York-London, 1970. MR0268655 64

[11] Jacek Graczyk and Stas Smirnov, Collet, Eckmann and Hölder, Invent. Math. 133 (1998), no. 1, 69-96, DOI 10.1007/s002220050239. MR1626469 166

[12] Bogusława Karpińska, Area and Hausdorff dimension of the set of accessible points of the Julia sets of $\lambda e^{z}$ and $\lambda \sin z$, Fund. Math. 159 (1999), no. 3, 269-287. MR1680622 162

[13] N. G. Makarov, Fine structure of harmonic measure, Algebra i Analiz 10 (1998), no. 2, 1-62; English transl., St. Petersburg Math. J. 10 (1999), no. 2, 217-268. MR1629379 636

[14] R. Daniel Mauldin and Mariusz Urbański, Dimensions and measures in infinite iterated function systems, Proc. London Math. Soc. (3) 73 (1996), no. 1, 105-154, DOI 10.1112/plms/s373.1.105. MR1387085 $7 2 \longdiv { 7 3 , 7 6 }$

[15] Volker Mayer and Mariusz Urbanski, Thermodynamical formalism for entire functions and integral means spectrum of tracts, Preprint, 2017.63, 66, 68, 74

[16] Ch. Pommerenke, Boundary behaviour of conformal maps, Grundlehren der Mathematischen Wissenschaften [Fundamental Principles of Mathematical Sciences], vol. 299, Springer-Verlag, Berlin, 1992. MR1217706 163, 64, 65, 66, 68, 69, 74

[17] Feliks Przytycki and Mariusz Urbański, Conformal fractals: ergodic theory methods, London Mathematical Society Lecture Note Series, vol. 371, Cambridge University Press, Cambridge, 2010. MR2656475 7376

[18] Lasse Rempe-Gillen, Hyperbolic entire functions with full hyperbolic dimension and approximation by Eremenko-Lyubich functions, Proc. Lond. Math. Soc. (3) 108 (2014), no. 5, 11931225, DOI 10.1112/plms/pdt048. MR3214678 64

[19] P. J. Rippon and G. M. Stallard, Dimensions of Julia sets of meromorphic functions with finitely many poles, Ergodic Theory Dynam. Systems 26 (2006), no. 2, 525-538, DOI 10.1017/S0143385705000544. MR2218773 162

[20] Gwyneth M. Stallard, The Hausdorff dimension of Julia sets of entire functions, Ergodic Theory Dynam. Systems 11 (1991), no. 4, 769-777, DOI 10.1017/S0143385700006477. MR.1145621 62

[21] Gwyneth M. Stallard, The Hausdorff dimension of Julia sets of entire functions. II, Math. Proc. Cambridge Philos. Soc. 119 (1996), no. 3, 513-536, DOI 10.1017/S0305004100074387. MR 1357062 62

[22] Gwyneth M. Stallard, Dimensions of Julia sets of transcendental meromorphic functions, Transcendental dynamics and complex analysis, London Math. Soc. Lecture Note Ser., vol. 348, Cambridge Univ. Press, Cambridge, 2008, pp. 425-446, DOI 10.1017/CBO9780511735233.017. MR2458811

[23] Anna Zdunik, Parabolic orbifolds and the dimension of the maximal measure for rational maps, Invent. Math. 99 (1990), no. 3, 627-649, DOI 10.1007/BF01234434. MR1032883 174

UFR de Mathématiques, UMR 8524 du CNRS, Université de Lille, 59655 Villeneuve D'Ascq Cedex, France

Email address: volker.mayer@univ-lille.fr

$U R L$ : math.univ-lille1.fr/ mayer 